\title{
ASPECTOS MACROSCÓPICOS DOS OVÁRIOS DE MATRIZES SUÍNAS, ORIUNDAS DE GRANJAS DA MICRORREGIÃO DE RIO VERDE-GO E DESCARTADAS PARA ABATE POR MOTIVOS DIVERSOS
}

\author{
(Macroscopic aspects of sow ovaries, natural from swine granges of \\ Rio Verde-GO and culling for several causes)
}

\author{
MOREIRA, F.'; PILATI, C. ${ }^{2}$; REIS, R.N. ${ }^{3}$; DICK, W. ${ }^{3}$; SOBESTIANSKY, J. ${ }^{4}$ \\ 'Mestre em Medicina Veterinária pela EV/UFG, a2fm@cav.udesc.br (Fone: (49)3223-4537); \\ ${ }^{2}$ Professor Doutor do Departamento de Patologia Animal da UDESC; \\ ${ }^{3}$ Alunos de Graduação de Medicina Veterinária CAV/UDESC; \\ ${ }^{4}$ Departamento de Medicina Veterinária da EV/UFG, Campus II Samambaia, Cx :131.
}

\begin{abstract}
There are many known causes for culling of sows in a herd, with reproductive problems standing as one of the major ones. In this scenary, replacement rates of $39-40 \%$ are considered acceptable in the pig industry. This study aimed to determine the relationship between functional ovarian condition in reproductively mature swine females and causes for culling. Hybrid Large White $x$ Landrace females from 10 pig farms in Rio Verde-GO, slaughtered during the period from March to July 2002 , had their ovaries analyzed by macroscopic examination and weighing. Decrease in production, advanced age, and low body condition score were the most common reasons for culling. From 330 pairs of ovaries analyzed under macroscopic examination, $90.6 \%$ were from cyclic females both on the follicular $(46.4 \%)$ or the luteal (44.2\%) phases, $7.6 \%$ had ovarian cysts, and $1.82 \%$ were in anestrus. The mean weight of ovaries was $8.8 \mathrm{~g}$, with females with cystic ovaries or in anestrus having the heaviest $(31.9 \mathrm{~g})$ and the lightest ovaries $(3.7 \mathrm{~g})$. A total of $98.8 \%$ of all animals had ovaries showing normal cyclicity, despite the decrease in production. These results showed no associations between the difference in ovary weight and stages of the estrous cycle, and between causes of culling and functional conditions of the ovaries.
\end{abstract}

Key-words: sow, reproduction; ovaries; culling.

RESUMO - Muitas são as causas já identificadas que determinam o descarte de matrizes suínas, onde as principais são de ordem reprodutiva, admitindo-se como aceitável uma taxa de reposição em granjas comerciais de $39-40 \%$ ao ano. O presente estudo foi realizado com o objetivo de determinar a relação entre a condição funcional encontrada nos ovários de matrizes suínas em produção com a causa de descarte destas fêmeas. Foram avaliados, através de exame macroscópico e pesagem, ovários de fêmeas mestiças Large Withe $x$ Landrace, descartadas de 10 granjas na microrregião de Rio Verde-GO no período de março a julho de 2002. As causas de descarte das fêmeas amostradas em sua maioria foram devido a baixa produtividade, a idade avançada e o mau escore corporal. No exame macroscópico pós-morte dos 330 pares de ovários avaliados destas fêmeas, foi observado que $46,36 \%$ estavam ciclando na fase folicular, $44,24 \%$ na fase lútea, $7,58 \%$ apresentavam cistos ovarianos e 1,82\% foram classificados como em estado de anestro. O peso médio dos ovários foi de $8,75 \mathrm{~g}$, sendo que aqueles que possuíam cistos foram os mais pesados $(31,94 \mathrm{~g})$ e os mais leves foram aqueles em estado anéstrico com $3,74 \mathrm{~g}$. Com base nestes dados observamos que $98,18 \%$ dos ovários avaliados estavam ciclando normalmente, apesar das fêmeas estarem apresentando queda na sua produção. Os resultados sugerem que não há nenhuma associação entre a diferença de peso apresentada pelos ovários direito e esquerdo, e as fases do ciclo estral. Relacionando as causas de descarte com a condição funcional dos ovários observou-se que não houve nenhuma dependência entre as duas variáveis.

Palavras-chave: fêmea suína; reprodução; ovários; descarte.

\section{Introdução}

Granjas manejadas dentro de um intenso fluxo de produção, trabalham com taxas anuais de descarte elevadas entre 35 e $50 \%$, onde a meta recomendada é de $40 \%$, sendo $35-36 \%$ para descarte e $3-5 \%$ para mortalidade. Muitas destas fêmeas são descartadas por problemas irreais, que podem ser oriundos de falhas humanas durante sua determinação, levando a um aumento na taxa de reposição, diminuição da produtividade e, conseqüentemente, aumento dos dias não produtivos e custos de produção (DIEHL et al. 2003).

Por outro lado, grandes unidades de reprodução de suínos, intensificação e complexidade tecnológica têm produzido novos tipos de problemas reprodutivos, bem como exacerbando formas antigas. $O$ tipo e o padrão dos distúrbios de fertilidade podem ser identificados a partir do material de abate avaliado e associados às causas de descarte, para deste modo estimar as perdas econômicas da criação (HEINONEN et al., 1998). Não são raros os casos em que, mediante o emprego do 
estudo isolado, por ocasião do abate dos órgãos genitais femininos, há evidência dos reais motivos que desencadeiam o transtorno reprodutivo em exame como causa de descarte de fêmeas suínas enviadas ao abate (ACLAND, 1998; VARGAS et al., 2005). A investigação e o controle das falhas reprodutivas podem ser instalados em granjas através do monitoramento dos diversos passos envolvidos na reprodução (FRANCESCHINI, 2001), pois mesmo em criações bem orientadas, as causas de natureza reprodutiva destacam-se entre os principais motivos de descarte de fêmeas suínas para o abate (CARVALHO, 1990, FRANCHESCHINI, 2001, TUMMARUK et al, 2001). Conforme TUMMARUK et al. (2001) descreveram, dentre o descarte por razões reprodutivas, destacamse fêmeas encontradas vazias após a cobrição, não demonstração de sinais de estro, descarga vulvar, repetição de cio, pequeno tamanho da leitegada e longo intervalo desmame-estro.

O exame dos ovários é peça fundamental para determinar o estágio do ciclo estral, e a avaliação das suas mudanças cíclicas normais é absolutamente indispensável para a interpretação e reconhecimento de possíveis anormalidades (BANKS, 1991). Assim, a avaliação dos órgãos do aparelho reprodutor por virtude do abate é sempre recomendada, pois o achado de porcas apresentando ovários cíclicos pode ser um indicativo de falhas no manejo de detecção do estro (VARGAS et al., 2005; KNOX, 2005). Dessa forma, a não detecção do retorno ao estro após cobertura pode levar a situação de fêmeas serem consideradas gestantes e, sendo estas transferidas para locais com condições desfavoráveis à detecção, dificulta o diagnóstico de retorno ao estro. (VARGAS et al., 2005)

O objetivo deste trabalho foi determinar a relação entre a condição funcional encontrada nos ovários de matrizes suínas em produção com a causa de descarte destas fêmeas.

\section{Material e Métodos}

O estudo envolveu 10 unidades de produção de leitões, de uma única empresa, localizadas na região de Rio Verde-GO. Cada unidade possuía em média 1100 matrizes em produção, mestiças Large Withe $x$ Landrace, das quais foram selecionadas 330 fêmeas, todas descartadas para abate em decorrência de inadequada performance reprodutiva. O estudo foi desenvolvido durante o período de março a julho de 2002. As causas de descarte de cada fêmea foram registradas de acordo com as informações recebidas pelos funcionários destas granjas. Eram abatidas em média 80 matrizes em produção descartadas/dia, sendo que destas retirava-se ao acaso uma amostragem de 12 a 15 fêmeas, independente da procedência. De acordo com os alvos de produtividade preconizados para este sistema intensivo de produção de leitões (SPL), fêmeas que não atingirem pelo menos 30 leitões nascidos/totais em três partos ou ainda, fêmeas com produção decrescente, isto é, se em dois partos consecutivos os nascidos totais forem menores que a média da granja, devem ser descartadas por baixa produtividade.

\section{QUADRO 1 -CRITÉRIOS PARA CLASSIFICAÇÃO E INTERPRETAÇÃO DOS ACHADOS OVARIANOS.}

\begin{tabular}{|l|l|}
\hline \multicolumn{1}{|c|}{ Características ovarianas } & \multicolumn{1}{c|}{ Classificação/interpretação } \\
\hline $\begin{array}{l}\text { Presença de folículos, corpos lúteos e corpos } \\
\text { hemorrágicos, com prevalência de folículos }\end{array}$ & Grau OF - ovários ciclando, fase folicular \\
\hline $\begin{array}{l}\text { Presença de folículos, corpos lúteos e corpos } \\
\text { hemorrágicos, com prevalência de corpos lúteos }\end{array}$ & Grau OL - ovários ciclando, fase lútea \\
\hline $\begin{array}{l}\text { Presença de cistos foliculares ou luteínicos, } \\
\text { únicos ou múltiplos }\end{array}$ & Grau 1 - cistos \\
\hline Ausência de folículos e corpos lúteos maduros & Grau 2 - estado de anestro \\
\hline
\end{tabular}

Fonte: Adaptado de MARTINAT-BOTTÉ et al. (2000); MORÉS et al. (2000).

Cada amostra de ovário recebia um número de identificação. Imediatamente após a colheita, os ovários foram avaliados macroscopicamente, fotografados e pesados em balança de precisão com sensibilidade de $0,01 \mathrm{~g}$ marca Acculab®. As informações sobre a classificação macroscópica e pesagem dos ovários foram anotadas em fichas individuais. A avaliação macroscópica dos ovários para determinar seu estado reprodutivo, foi realizada de acordo com a classificação adaptada de MARTINAT-BOTTÉ et al. (2000); MORÉS et al. (2000); SOBESTIANSKY et al. (2001), conforme o QUADRO1.

O teste utilizado para a análise estatística foi o teste de $x^{2}$ (Qui-quadrado). Nestas unidades as instalações, o manejo, a nutrição, o programa sanitário e de biossegurança eram idênticos, de tal forma que a única diferença entre elas restringia-se aos funcionários.

\section{Resultados e Discussão}

A baixa produtividade foi a causa de descarte mais freqüente das matrizes suínas mantidas em sistema intensivo de produção de leitões (SPL). Do total de 330 fêmeas suínas avaliadas $41,52 \%$ foram descartadas por baixa produção. A segunda maior causa para 0 descarte, atingindo $11,21 \%$, foi a idade avançada, ou seja, fêmeas com mais de seis partos. Constatou-se que $7,88 \%$ das 330 matrizes foram descartadas devido a condição escore corporal ruim, categoria muito magra ou magra, ou seja, fêmeas com ossos das costelas 
Aspectos macroscópicos dos ovários de matrizes suínas, oriundas de granjas da microrregião...

proeminentes e pouca profundidade abdominal, o que de acordo com PRUNIER e QUESNEL (2002) e FOXCROFT (2002) isto pode influenciar negativamente no crescimento folicular, provocar decréscimo do padrão ovulatório, atraso na entrada à puberdade e no retorno ao cio pós-desmame. Problemas locomotores perfizeram o total de $12,43 \%$ e, destes, $7,58 \%$ e $4,85 \%$ das fêmeas foram eliminadas por problemas de aprumos e problemas de casco, respectivamente. SOBESTIANSKY et al. (1999) relataram que o aparecimento das lesões nos cascos provoca incômodo e dor nas matrizes, favorecendo o esmagamento de leitões, o emagrecimento, a hipoagalaxia, o abortamento, a infertilidade e/ou o aumento na taxa de descarte. Dentro das causas reprodutivas analisadas neste estudo, o retorno ao estro duas vezes consecutivas em período regular ou irregular foi o motivo pelo qual 17 matrizes $(5,15 \%)$ foram descartadas. 0 intervalo de repetição do cio fornece informações para classificar o retorno ao estro como regular ou irregular (TUMMARUK, et al. 2001). Um percentual maior de fêmeas $(4,85 \%)$ que foram descartadas por aborto foi registrado em contraponto com as que apresentaram falsa gestação (3,64\%). Segundo KOKETSU et al.
(1997), isto indica que a produtividade de um rebanho depende igualmente do diagnóstico de estro e outros testes para o estado de prenhez, tal como ultrasonografia, avaliação visual e observação de abortos. A mortalidade embrionária ocorrendo de 25 a 30 dias de gestação pode resultar em pseudogestação, porque o corpo lúteo continua a produzir progesterona até 3 meses após a morte dos fetos. O descarte por anestro atingiu um percentual de 3,03\% de todas as causas de descarte. Para fêmeas que foram descartadas devido a lesões na escápula, articulações, membros anteriores e posteriores que não lesões nos cascos o percentual atingiu $3,33 \%$. Estas matrizes sofreram lesões nas gaiolas as quais impediam a cobertura, devido ao desconforto ou devido à dor causada pela lesão. A descarga vulvar foi a causa de descarte de menor freqüência $(2,73 \%)$. Neste caso as fêmeas apresentavam secreções vulvares ou corrimento, as quais, de acordo com SOBESTIANSKY et al. (1999), constituem um sinal clínico que pode se apresentar de forma discreta e profusa, com aspecto variando de muco purulento a hemorrágico. A TABELA 1 apresenta a freqüência encontrada das causas de descarte das matrizes suínas.

TABELA 1 - FREQÜÊNCIA DAS CAUSAS DE DESCARTE DAS MATRIZES SUÍNAS MANTIDAS EM SISTEMA INTENSIVO DE PRODUÇÃO DE LEITÕES LOCALIZADOS NA MICRORREGIÃO DE RIO VERDE - GO NO PERÍODO DE MARÇO A JULHO 2002.

\begin{tabular}{lcc}
\hline \multicolumn{1}{c}{ Causas de Descarte } & Número de Fêmeas & $\%$ \\
\hline Baixa produtividade & 137 & 41.52 \\
Idade avançada & 37 & 11,21 \\
Escore corporal ruim & 26 & 7,88 \\
Problema de aprumo & 25 & 7,58 \\
Repetição de estro & 17 & 5,15 \\
Problema de casco & 16 & 4,85 \\
Aborto & 16 & 4,85 \\
Falsa gestação & 12 & 3,64 \\
Machucada & 11 & 3,33 \\
Anestro & 10 & 3,03 \\
Descarga vulvar $_{\text {Outras causas }^{2}}$ & 09 & 2,73 \\
Total & 14 & 4,24 \\
\hline
\end{tabular}

'Lesões na escápula, articulações, membros anteriores e posteriores (de acordo com as informações cedidas pela assistência técnica);

${ }^{2}$ Mastite, lleíte, Prolapso retal e uterino .

TABELA 2 - NÚMERO E PERCENTUAL DE FÊMEAS ESTUDAS NO MUNICÍPIO DE RIO VERDE - GO DE ACORDO COM A CONDIÇÃO FUNCIONAL DE SEUS OVÁRIOS.

\begin{tabular}{lcc}
\hline Condição funcional dos ovários & $N^{\circ}$ Fêmeas & Percentual (\%) \\
\hline Grau OF Ciclando-fase folicular & 153 & 46,36 \\
Grau OL Ciclando-fase lútea & 146 & 44,24 \\
Grau 1 Cisto ovariano & 25 & 7,58 \\
Grau 2 Estado de anestro & 6 & 1,82 \\
Total de fêmeas & 330 & - \\
\hline
\end{tabular}


Conforme pode ser verificado na TABELA 2, no exame macroscópico pós-morte dos 330 pares de ovários para identificação da sua condição funcional, foi observada a presença de ovários ciclando na fase folicular em 153 fêmeas (46,36\%), ovários ciclando na fase lútea em 146 fêmeas $(44,24 \%)$, cistos ovarianos em 25 fêmeas $(7,58 \%)$ e em seis casos $(1,82 \%)$ os ovários foram classificados como em estado de anestro. Considerando que a situação fisiológica dos ovários classificados como Grau OF e Grau OL é considerada como fazendo parte do ciclo estral normal verifica-se na TABELA 2 que 299 matrizes descartadas (90,60\%) estavam em atividade cíclica normal. Com relação ao exame macroscópico pós-morte dos ovários EINARSSON et al. (1982) e DALIN et al. (1997) também verificaram que a maioria das fêmeas descartadas envolvidas em seus estudos apresentavam atividade ovariana, porém em percentuais mais baixos, ou seja, 38,0 e $69,0 \%$, respectivamente. De acordo com FOXCROFT (2002), é possível que o perfil estral e ovulatório da fêmea suína seja influenciado pela ordem do parto ou pela redução nas reservas corporais de energia, caracterizada por redução na espessura de toucinho. Dos ovários avaliados $7,58 \%$ foram classificados como ovários císticos por possuírem algum tipo de cisto em um ou em ambos ovários. Os ovários cistícos encontrados apresentavam tamanho superior ao dos folículos pré-ovulatórios ou corpos lúteos maduros, e eram preenchidos com fluído. Em porcas podem estar presentes cistos individuais em conjunto ao corpo lúteo, encontrados em um ou ambos ovários. Podem ser cistos foliculares, luteínicos e hemorrágicos, no entanto, neste estudo, somente foram encontrados cistos foliculares e luteínicos. Foi observado que das 25 fêmeas com cistos ovarianos 17 (68\%) apresentavam ovários com cistos múltiplos e que apenas $8(32 \%)$ destas possuíam cistos simples ou individuais em seus ovários. Quando existe a formação de múltiplos cistos, isto significa que ocorreu distúrbio endócrino como uma diminuição de LH ou alteração na relação FSH/LH. Quando se desenvolve cisto individual não se pode dizer que há transtornos hormonais, mas pode-se afirmar que a causa está relacionada com o desenvolvimento folicular, pois na maior parte das vezes o número de folículos que está em desenvolvimento é normal (SCHNURRBUSCH e SCHARFE, 1991; NASCIMENTO e SANTOS, 2002). O efeito do estresse também é uma das causas que aumentam a incidência de cistos ovarianos, fato este que implica na redução da fertilidade ou infertilidade completa (SCHARFE e SCHNURRBUSCH, 1990). CASTAGNA et. al.(2004) demonstraram que o aparecimento de cistos influenciou a taxa de retorno ao estro, taxa de parto e taxa de fêmeas encontradas vazias ao parto, mas não o tamanho da leitegada. Os ovários pertencentes à categoria dos ovários em estado de anestro ou atrofiados foram registrados em baixa porcentagem $(1,82 \%)$. Os ovários em estado de anestro, observados pela avaliação macroscópica, obtiveram uma parcela muito inferior ao que era esperado, já que naquelas matrizes que foram descartadas por apresentarem anestro clínico não foi confirmado em nenhum caso ovário em estado de anestro. KNOX (2005) descreveu que a avaliação e visualização dos ovários de porcas prova ser um ótimo método para auxiliar as decisões de manejo como o descarte destas fêmeas das criações de suínos, por exemplo, a identificação de ovários anéstricos em leitoas pré-púberes e porcas após o desmame. Sendo assim, LUCIA et al. (2000) citam que nem sempre é possível observar claramente, menor eficiência produtiva anual, em granjas com altas taxas de descarte por problemas reprodutivos, por isso o acompanhamento ao abate pode ser útil, trazendo informações importantes para comparar as razões dadas para o descarte de matrizes suínas e status fisiológico do trato reprodutivo.

Conforme mostra a FIGURA 1, foram pesados 330 pares de ovários, sendo o peso médio de $13,30 \mathrm{~g}$, com uma variação média geral de $3,70 \mathrm{~g}$ (ovários em anestro) a $31,94 \mathrm{~g}$ (ovários com presença de cisto). Por outro lado, considerando apenas os ovários ciclando normalmente (Grau OF + Grau 0L), o peso médio respectivo foi de $8,75 \mathrm{~g}$, valor que se encontra abaixo do registrado por WRATHALL, (1980) e McENTEE (1990) os quais estabeleceram que ovários sexualmente maduros possuem peso médio variando entre de $10-20 \mathrm{~g}$.

$\mathrm{Na}$ FIGURA 1 observa-se que dentro da categoria dos ovários que estavam ciclando, os pertencentes à fase lútea foram mais pesados (média de $10,0 \mathrm{~g}$ ) que os ovários em fase folicular que revelaram média de $7,5 \mathrm{~g}$. PRIEDKALNS (1982) e BANKS (1991) justificam tal fato quando na formação do corpo lúteo há o acúmulo de lipídios marcando a transição das células da granulosa para células granulosa-luteínicas, a invasão desta região por células do estroma e por vasos resultando na deposição de fibras reticulares convertendo esta estrutura em glândula altamente vascularizada e bastante densa pela composição quase que completa de tecido luteínico, ao contrário dos folículos que são estruturas compostas por pequenas lacunas ou fendas intercelulares preenchidas por fluido o "líquido folicular", sendo estruturas bem menos densas, portanto, mais leves. 
Aspectos macroscópicos dos ovários de matrizes suínas, oriundas de granjas da microrregião...

\section{FIGURA 1 - PESO MÉDIO E CONDIÇÃO FUNCIONAL DOS OVÁRIOS DE 330 MATRIZES, ORIUNDAS DE GRANJAS} DA MICRORREGIÃO DE RIO VERDE-GO NO ANO DE 2002.

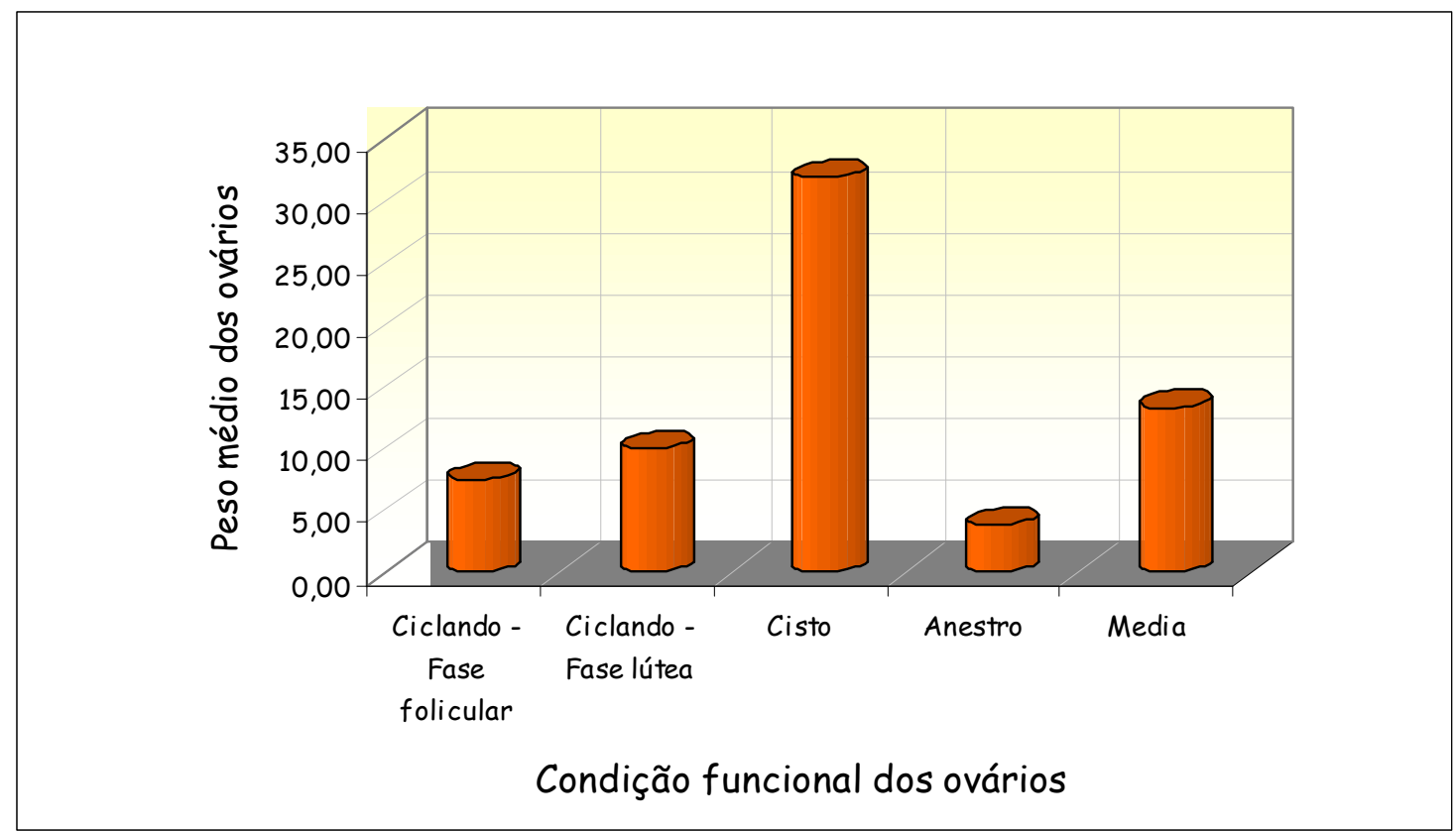

O peso médio dos ovários determinado neste estudo foi inferior ao descrito na literatura, portanto pode-se dizer que estes resultados se comportaram desta forma devido ao fato dos ovários que estavam ciclando na fase folicular, apresentarem-se em maior freqüência $46,36 \%$, pois ovários nesta fase são relativamente mais leves que aqueles que estavam em atividade cíclica na fase lútea. Verificou-se, ainda, que os pesos médios dos ovários variam em função de sua localização anatômica (direita e esquerda).
Os ovários esquerdos pesaram, em média, 0,95g a mais que os ovários direitos (FIGURA 2). Independente desta diferença de peso observada, conclui-se que não exista nenhuma associação entre o peso dos mesmos e as fases do ciclo estral, pois não são as estruturas funcionais que compõem os ovários que determinam o seu peso, e sim, o tecido pelo qual são compostos.

De acordo com a análise estatística baseada nos resultados do teste de $x^{2}$ (Qui-quadrado) não existe relação entre as variáveis estudadas.

FIGURA 2 - PESOS MÉDIOS DOS OVÁRIOS DIREITO E ESQUERDO DE ACORDO COM SUA CONDIÇÃO FUNCIONAL, ORIUNDAS DE MATRIZES SUÍNAS DA MICRORREGIÃO DE RIO VERDE - GO NO ANO DE 2002.

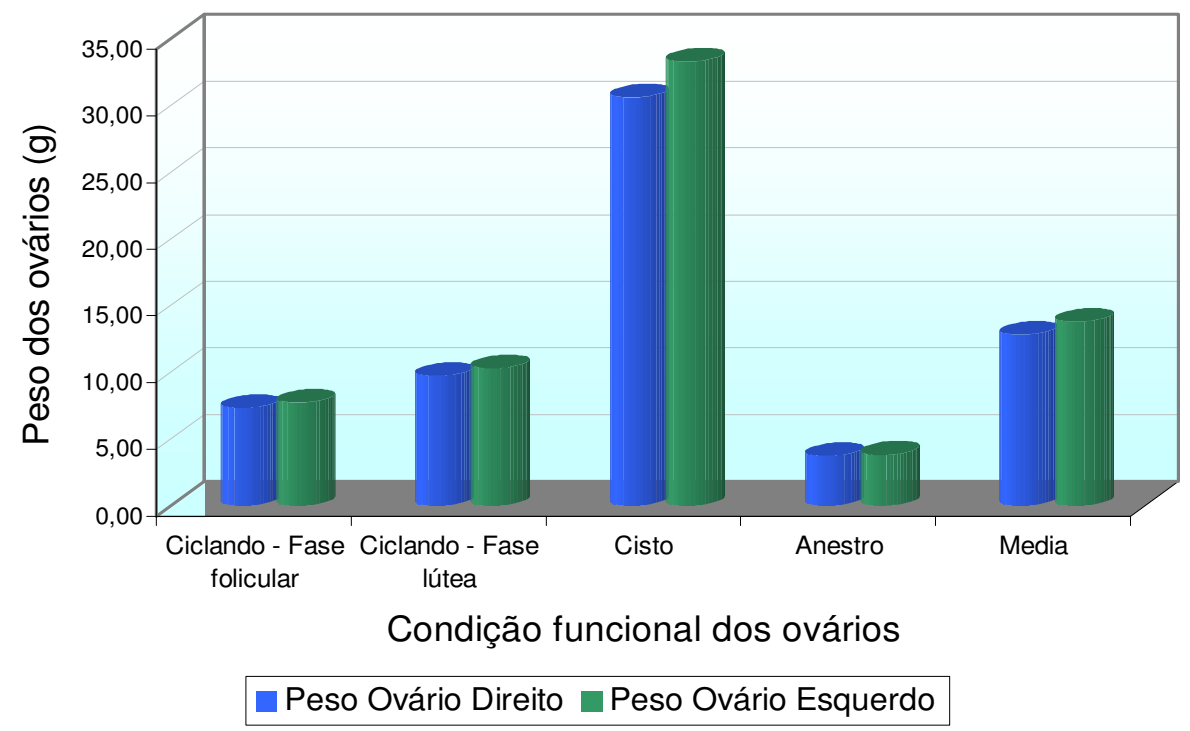




\section{Conclusões}

- A baixa produtividade, a idade avançada e o escore corporal ruim foram as causas mais freqüentes de descarte de matrizes nos rebanhos.

- A grande maioria das matrizes descartadas apresentou função ovariana normal, pois os ovários destas fêmeas apresentaram-se em atividade cíclica na fase folicular ou na fase lútea.

- As causas de descarte não mostraram qualquer relação com a condição funcional dos ovários das matrizes suínas em estudo.

\section{Referências}

ACLAND, H.M. Sistema reprodutor feminino. In: THOMSON, R. G. Patologia veterinária especial. São Paulo: Manole , 1998, p.736.

BANKS, W.J. Histologia Veterinária Aplicada. 2ed. São Paulo: Manole, 1991, p. 565-89.

CARVALHO, L.F.O.S. Investigação clínica, anatomopatológica e citogenética de fêmeas suínas com transtornos reprodutivos. 1990. 95p. Tese (Doutorado). Faculdade de Medicina Veterinária e Zootecnia. Universidade Estadual Paulista.

CASTAGNA, C.D.; PEIXOTO, C.H.; BORTOLOZZO, F.P.; WENTZ, I; BORCHARDT NETO G.; RUSCHEL, F. Ovarian cysts and their consequences on the reproductive performance of swine herds. Animal Reproduction Science. v.81, p.115-123, 2004.

DALIN, A.M.; GIDLUND, K.; ELIASSON, S. L. Post-morten examination of genital organs from sows with reproductive disturbances in a sow-pool. Acta Veterinaria Scandinavica. v.38, n. 3, p. 253-62, 1997.

DIEHL, G.N.; COSTI, G.; VARGAS, A.J.; RICHTER, J.B.; LECZNIESKI, L.F.; BORTOLOZZO, F.P.; BERNARDI, M.L.; WENTZ, I. Monitoramento ovariano ao abate de leitoas descartadas por anestro ou estro atípico. Archives of veterinary Science, v.8, n 1, p. 121-125, 2003.

EINARSSON, S.; LUNDEHEIM, N.; MARTINASSON, K.; PERSSON, N.; PERSSON, I. Post-mortem examination of the genital organs of culling sows from one large herd with relation to fertility data. In: Internaestronal Pig Veterinary Society. 7., Mexico, 1982. Proceedings. p. 211.

FOXCROFT, G.R. Reproductive management for the new millennium. In: Congresso Latino Americano de Suinocultura. Anais. Foz do Iguaçu-PR. p. 86-94. 2002.

FRANCESCHINI, P.H. Falhas reprodutivas em suínos. In: Simpósio Sobre Manejo e Nutrição de Aves e Suínos e Tecnologia da Produção de Rações. Colégio Brasileiro de Nutrição Animal. Campinas, 2001, p. 227-236.

HEINONEN, M.; LEPPAVUORI, A.; PYORALLA, S. Evaluation of reproductive failure of female pigs based on slaughterhouse material and herd record survey. Animal Reproduction Science. v.56, n.3, p.235-44, set., 1998.
KNOX, R.V. Real-time ultrasound asssesment of reproductive status in pigs. Swine reproductinon. p.16-25. 2005.

KOKETSU, Y.; DIAL, G.D.; KING, V.L. Returns to service after mating and removal of sows for reproductive reasons from comercial swine farms. Theriogenology. v. 47, n.7, p. 1347-63, 1997.

LUCIA, T.; CORRÊA, M.N.; DESCHAMPS, J.C. Tópicos em suinocultura. Pelotas: JH Barbacha, 2000, 196p.

MARTINAT-BOTTÉ, F.; RENAVO, G.; MADE, F.; COSTIOU, P.; TERQUI, M. Ultrasonography and reproduction in swine. Paris: INRA, 2000, p.103.

McENTEE, K. Reproductive pathology of domestic mammals. San Diego: Academic Press, CA, 1990, 401p.

MORÉS, N.; SOBESTIANSKY, J.; LOPEZ, A. Avaliação patológica de suínos no abate: manual de identificação. Brasília: EMBRAPA comunicação para transferência de tecnologia, 2000, 40p.

NASCIMENTO, E.F.; SANTOS, L.R. Patologia da reprodução dos animais domésticos. 2ed. Rio de janeiro: Guanabara Koogan, 2002, p. 22-29.

PRIEDKALNS, J. Sistema reprodutor feminino. In: DELLMANN; H., BROWN, E. Histologia Veterinária. Rio de Janeiro: Guanabara Koogan S. A, 1982, p. 255-79.

PRUNIER, A.; QUESNEL, $H$. Influence of the nutricional status on ovarian development in female pigs. Animal reproduction science. v.60-61, p. 185-197, 2002.

SCHARFE, S.; SCHNURRBUSCH, U. Occurrence and causes of cystic ovaries in sows. Karl Marx Universitat. v.39, n.3, p.280-292, 1990.

SCHNURRBUSCH, U.; SCHARFE, S. Zum vorkommen verschiedener formen der ovarialzysten des schweines unter besonderer berücksichtigung ihres einflusses auf den zyklusverlauf. Tierärztliche Praxis, v.19, p.635-43, 1991.

SOBESTIANSKY, J.; BARCELLOS, D.E.S.N.; MORES, N.; OLIVEIRA, S.J.; CARVALHO, L.O.S.; MORENO, M.A.; ROEHE, P.M. Clínica e patologia suína. 2ed., Goiânia, 1999, 464p.

SOBESTIANSKY, J.; MATOS, M.P.C.; SOUZA, C.M. Monitoria do aparelho genital e urinário. In: SOBESTIANSKY, J. Monitoria patológica de suínos em matadouros. Goiânia, 2001, 52p.

TUMMARUK, P.; LUNDEHEIM, N.; EINARSSON, S.; DALIN, A.M. Repeat breeding and subsequent reproduvtive performance in Swedish Landrace end Swedish Yorkshire sows. Animal reproduction science. v.67, p. 267-280, 2001.

VARGAS, A.J.; WENTZ, I.; BORTOLOZZO, F. Desempenho de fêmeas suínas após apresentarem falhas reprodutivas. In: V Seminário Internacional de Aves e Suínos - Ave Sui, Anais. Florianópolis-SC, p. 256-63. 2005.

WRATHALL, A.E. Ovarian disorders in the sow. Vet. Bull. Farnham Royal, v.50, n.4, p. 253-72, 1980.

Recebido para publicação: $13 / 09 / 2006$ Aprovado: 